\title{
A CTX-M extended-spectrum $\beta$-lactamase in Pseudomonas aeruginosa and Stenotrophomonas maltophilia
}

Extended-spectrum $\beta$-lactamases (ESBLs) are an important cause of bacterial resistance throughout the world (Paterson \& Bonomo, 2005). CTX-M ESBLs were first reported in 1986 (Matsumoto et al., 1988) and their dissemination among bacterial species and in various parts of the world during the past decade is of growing concern (Bonnet, 2004). Recent studies indicate that the CTX-M enzymes predominate among the ESBLs of community strains (Pitout et al., 2005). CTX-M ESBLs probably originated from Kluyvera species and these enzymes are mostly found in members of the Enterobacteriaceae. Recently, the emergence of these enzymes has been reported in Acinetobacter baumannii (Nagano et al., 2004).

\section{Pseudomonas aeruginosa and}

Stenotrophomonas maltophilia are opportunistic pathogens responsible for nosocomial infections. Phenotypic detection of ESBLs in these species is complex for various reasons. In $P$. aeruginosa, false-negative results may occur due to chromosome-encoded $\beta$-lactamases, such as the AmpC enzymes, which may be overexpressed, due to the simultaneous presence of metallo-enzymes with carbapenem-hydrolysing activities (the IMP and VIM series), or due to combined mechanisms of resistance, such as impermeability and efflux (Weldhagen et al., 2003). In S. maltophilia, clavulanate tests may produce false-positive results via inhibition of the L2 chromosome-encoded $\beta$-lactamase (Walsh et al., 1997).

In this study, we describe the presence of CTX-M-1 $\beta$-lactamase in two clinical bacterial strains of $P$. aeruginosa and $S$. maltophilia.

P. aeruginosa and S. maltophilia strains were isolated during a prevalence study on ESBLs in May 2004 in Amsterdam at the microbiological laboratory of the Academic Medical Center (AMC). The P. aeruginosa strain was isolated from the sputum of a 21-year-old male cystic fibrosis outpatient. The S. maltophilia strain was isolated from the sputum of a hospitalized, male neonate at the Department of Neonatology at AMC. Neither patient showed any signs of invasive infection due to the presence of these strains. Antimicrobial regimens before admission were not documented and the patients were not receiving any antibiotic treatment at the time of isolation of these strains.

The strains were identified as $P$. aeruginosa and S. maltophilia using VITEK-2 (bioMérieux) and classical biochemical determination (routine determination at the microbiology laboratory). Species determination was confirmed by PCR and sequence analysis of the $16 \mathrm{~S}$ rRNA gene, using the generic primers $\mathrm{p} 515 \mathrm{~F}$ (5'-TGCCAGCAGCCGCGGTAA-3') and p13b (5'-AGGCCCGGGAACGTATTCAC3') (Relman et al., 1992).

Antibiotic susceptibilities were determined according to the guidelines of the National Committee for Clinical Laboratory Standards (NCCLS) with the disc diffusion method (NCCLS, 2005). ESBL production was detected using a combination of the double disc test and the combined disc test (DCDT) (N. al Naiemi, B. Duim, V. van der Veen, M. D. de Jong \& A. Bart, unpublished data), which included discs of ceftazidime, cefotaxime, cefpodoxime and cefepime placed around a disc containing amoxycillin plus clavulanate. The test was done at two distances between the discs: 30 and $20 \mathrm{~mm}$ (centre to centre). The DCDT also included a disc containing ceftazidime plus clavulanate and a disc containing cefoxitin for detection of AmpC production.

The presence of the ESBL genes $b l a_{\mathrm{SHV}}$, $b l a_{\text {TEM }}$ and $b l a_{\text {CTX-M }}$ was determined by PCR and sequence analysis using $b l a_{\mathrm{SHV}^{-}}$and $b l a_{\mathrm{TEM}^{-}}$-specific primers as described previously (al Naiemi et al., 2005) and primers CTX-MA-F1
(5'-CGYTTTSCRATGTGCAG-3') and CTX-MB-F2 (5'-ACCGCRATATCRTTGGT-3'), slightly modified from primers used by Bonnet et al. (2001). To prevent carry-over contamination, the Uracil system (Roche) was applied and ultrahigh-purity water was used as a negative control. The PCR conditions were identical to those described previously (al Naiemi et al., 2005). Both DNA strands were sequenced with the amplification primers and two additional internal $b l a_{\mathrm{SHV}}$ and $b l a_{\mathrm{TEM}}$ primers as described previously (al Naiemi et al., 2005).

The ESBL phenotype of the $P$. aeruginosa and $S$. maltophilia strains is shown in Fig. 1. As the $P$. aeruginosa isolate was sensitive to inhibition by clavulanate (Fig. 1), the action of OXA $\beta$-lactamases, which are frequently present in $P$. aeruginosa, is unlikely (Bradford, 2001). The susceptibility of this $P$. aeruginosa strain for cefoxitin is noteworthy. $P$. aeruginosa is usually resistant to cefoxitin, as it contains an inducible AmpC enzyme (Livermore, 1991). However, failure of cefoxitin to induce AmpC production in $P$. aeruginosa has been reported previously (Ramadan et al., 1995).

PCR and sequence analysis revealed the presence of the $b l a_{\mathrm{CTX}-\mathrm{M}-1}, b l a_{\mathrm{SHV}-1}$ and $b l a_{\mathrm{TEM}-116}$ genes in the $P$. aeruginosa strain and $b l a_{\mathrm{CTX}-\mathrm{M}-1}$ and $b l a_{\mathrm{SHV}-1}$ in the $S$. maltophilia strain. Of these $\beta$-lactamases, only CTX-M-1 has been shown to produce a transferable ESBL phenotype (Sirot et al., 1987).

To our knowledge, this is the first description of the presence of CTX-M ESBLs in $P$. aeruginosa and S. maltophilia. The CTX-M ESBLs provide these pathogens with an additional powerful resistance mechanism with potential serious clinical implications, including limitation of the therapeutic options.

It remains to be determined how the CTX-M-1 genes have disseminated to these 

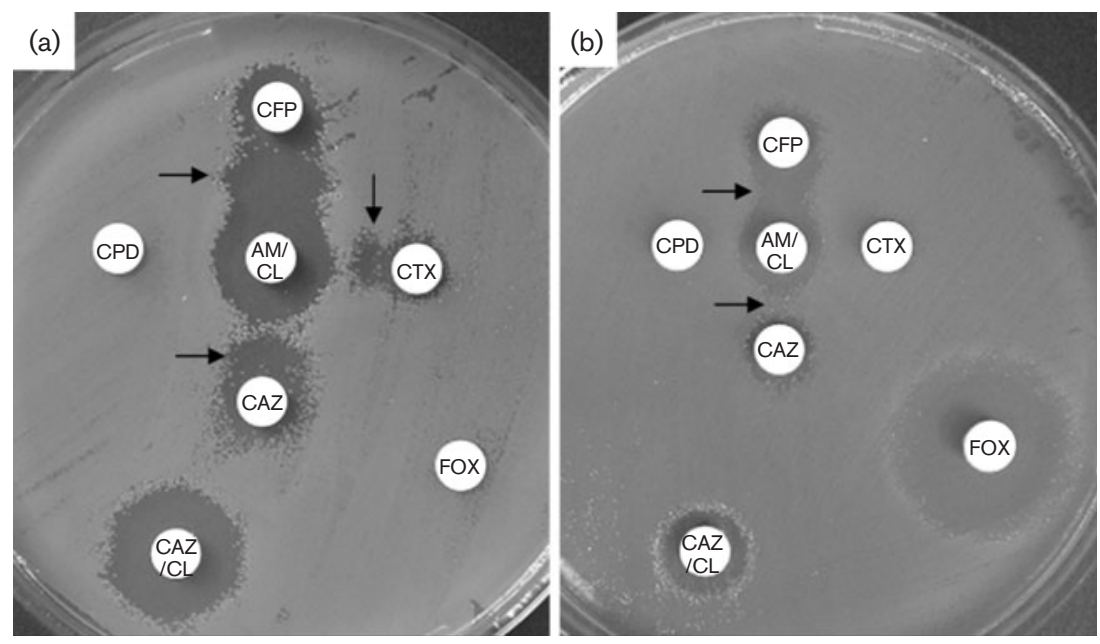

Fig. 1. Phenotypic detection of ESBL. (a) DCDT for S. maltophilia with CTX-M-1, at $30 \mathrm{~mm}$ distance between the discs (centre to centre). Production of ESBL is indicated by the synergy between the CAZ, CTX and CFP discs and the AM/CL disc (arrows). In addition, the $\mathrm{CAZ} / \mathrm{CL}$ zone was $\geqslant 5 \mathrm{~mm}$ larger than that of $C A Z$, indicating the production of ESBL. (b) DCDT for $P$. aeruginosa with $\mathrm{CTX}-\mathrm{M}-1$, at $20 \mathrm{~mm}$ distance between the discs (centre to centre). Synergy is evident between the CFP and $C A Z$ discs and the $A M / C L$ disc, indicating the production of ESBL. The CAZ/CL zone was $\geqslant 5 \mathrm{~mm}$ larger than that of $C A Z$, indicating the production of ESBL. CFP, Cefepime; CPD, cefpodoxime; $\mathrm{AM} / \mathrm{CL}$, amoxycillin/clavulanate; CTX, cefotaxime; CAZ, ceftazidime; FOX, cefoxitin; CAZ/CL, ceftazidime/clavulanate.

P. aeruginosa and S. maltophilia strains. The association of CTX-M $\beta$-lactamaseencoding genes with mobile elements such as the ISEcp 1 insertion element and integrons (Eckert et al., 2006) may facilitate the spread of $b l a_{\mathrm{CTX}-\mathrm{M}}$ genes among bacteria.

As phenotypic detection of ESBLs in nonfermenters is complicated and many clinical microbiological laboratories have no routine surveillance of ESBLs in nonfermenters, ESBLs may be underestimated and underreported in these strains. Therefore, P. aeruginosa and S. maltophilia may become hidden reservoirs for such ESBLs, as is already the case for OXA $\beta$-lactamases in $P$. aeruginosa.

\section{Acknowledgements}

We thank C. E. Visser and R. du Maine for their support in species determination.

\section{Nashwan al Naiemi, Birgitta Duim and Aldert Bart}

Department of Medical Microbiology, L1-244, Academic Medical Center, PO Box 22660, 1100 DD Amsterdam, The Netherlands

\section{Correspondence: Birgitta Duim (b.duim@amc.uva.nl)}

al Naiemi, N. A., Duim, B., Savelkoul, P. H. M., Spanjaard, L., de Jonge, E., Bart, A., Vandenbroucke-Grauls, C. M. \& de Jong, M. D. (2005). Widespread transfer of resistance genes between bacterial species in an intensive care unit: implications for hospital epidemiology. J Clin Microbiol 43, 4862-4864.

Bonnet, R. (2004). Growing group of extended-spectrum $\beta$-lactamases: the CTX-M enzymes. Antimicrob Agents Chemother 48, 1-14.

Bonnet, R., Dutour, C., Sampaio, J. L. M., Chanal, C., Sirot, D., Labia, R., De Champs, C. \& Sirot, J. (2001). Novel cefotaximase (CTX-M-16) with increased catalytic efficiency due to substitution Asp-240 $\rightarrow$ Gly. Antimicrob Agents Chemother 45, 2269-2275.

Bradford, P. A. (2001). Extended-spectrum $\beta$-lactamases in the 21st century:

characterization, epidemiology, and detection of this important resistance threat. Clin Microbiol Rev 14, 933-951.

Eckert, C., Gautier, V. \& Arlet, G. (2006). DNA sequence analysis of the genetic environment of various $b a_{\text {СTX-M }}$ genes. J Antimicrob Chemother 57, 14-23.

Livermore, D. M. (1991). $\beta$-Lactamases of Pseudomonas aeruginosa. Antibiot Chemother 44, 215-220.

Matsumoto, Y., Ikeda, F., Kamimura, T., Yokota, Y. \& Mine, Y. (1988). Novel plasmid-mediated $\beta$-lactamase from Escherichia coli that inactivates oxyiminocephalosporins. Antimicrob Agents Chemother 32, 1243-1246.

Nagano, N., Nagano, Y., Cordevant, C., Shibata, N. \& Arakawa, Y. (2004). Nosocomial transmission of CTX-M-2 $\beta$-lactamaseproducing Acinetobacter baumannii in a neurosurgery ward. J Clin Microbiol 42, 3978-3984.

NCCLS (2005). Performance standards for antimicrobial susceptibility testing; 15th informational supplement (M100-S15). Wayne,
PA: National Committee for Clinical Laboratory Standards.

Paterson, D. L. \& Bonomo, R. A. (2005). Extended-spectrum $\beta$-lactamases: a clinical update. Clin Microbiol Rev 18, 657-686.

Pitout, J. D. D., Nordmann, P., Laupland, K. B. \& Poirel, L. (2005). Emergence of

Enterobacteriaceae producing extended-spectrum $\beta$-lactamases (ESBLs) in the community. J Antimicrob Chemother 56, 52-59.

Ramadan, M. A., Tawfik, A. F. \& Shibl, A. M. (1995). Effect of $\beta$-lactamase expression on susceptibility of local isolates of Enterobacter cloacae, Serratia marcescens and Pseudomonas aeruginosa to $\beta$-lactam antibiotics.

Chemotherapy 41, 193-199.

Relman, D. A., Schmidt, T. M., MacDermott, R. P. \& Falkow, S. (1992). Identification of the uncultured bacillus of Whipple's disease. $N$ Engl J Med 327, 293-301.

Sirot, D., Sirot, J., Labia, R., Morand, A., Courvalin, P., Darfeuille-Michaud, A.,

Perroux, R. \& Cluzel, R. (1987). Transferable resistance to third-generation cephalosporins in clinical isolates of Klebsiella pneumoniae: identification of CTX-1, a novel $\beta$-lactamase. $J$ Antimicrob Chemother 20, 323-334.

Walsh, T. R., MacGowan, A. P. \& Bennett, P. M. (1997). Sequence analysis and enzyme kinetics of the L2 serine beta-lactamase from Stenotrophomonas maltophilia. Antimicrob Agents Chemother 41, 1460-1464.

Weldhagen, G. F., Poirel, L. \& Nordmann, P. (2003). Ambler class A extended-spectrum $\beta$-lactamases in Pseudomonas aeruginosa: novel developments and clinical impact. Antimicrob Agents Chemother 47, 2385-2392. 